\title{
A short term education intervention but not peer discussion improved vitality and pain in women with breast cancer
}

\section{Helgeson VS, Cohen S, Schulz R, et al. Long-term effects of educational and peer discussion group interventions on adjustment to breast cancer. Health Psychol 2001 Sep;20:387-92. \\ QUESTIONS: In women with breast cancer, are the positive effects of a short term education intervention maintained over time? Does a peer discussion intervention have positive effects $\geqslant 1$ year later?}

\section{Design}

Randomised (unclear allocation concealment; unclear if blinded) controlled trial with a mean follow up of 40 months.

\section{Setting}

Pittsburgh, Pennsylvania, USA.

\section{Patients}

312 women who were 27-75 years of age (mean age 48 y, 93\% Caucasian), had breast cancer (National Cancer Institute criteria stage I: 25\%; stage II: 69\%; and stage III: $6 \%)$, and had been treated by surgery $(68 \%$ lumpectomy, 32\% mastectomy) and adjuvant chemotherapy. Follow up was $83 \%$.

\section{Intervention}

Women were allocated to education $(\mathrm{n}=79)$, peer discussion $(\mathrm{n}=74)$, education plus peer discussion $(\mathrm{n}=82)$, or control ( $\mathrm{n}=77)$, approximately 3 months after diagnosis. Each group comprised 7 small groups of 8-12 women. Education consisted of 8 weekly 45 minute meetings in which an expert lectured on a topic (eg, nutrition), followed by a brief question and answer period. The goal of the education intervention was to provide expert information and enhance women's control over the illness experience. Peer discussion involved 8 weekly 60 minute meetings in which a master's level oncology social worker and nurse facilitated a group discussion on feelings and self disclosure. Group members determined the content of the discussion. The combined intervention consisted of weekly meetings that began with the educational lecture and were followed by a discussion group that lasted approximately 1 hour.

\section{Main outcome measure}

Health related quality of life (SF-36 Health Survey).

\section{Main results}

Analysis was by intention to treat. At follow up, the education group had greater vitality (mean score $62.7 v$ $57.3, \mathrm{p}=0.01$ ), less bodily pain (mean score $81.8 v 75.7$, $\mathrm{p}=0.02$ ), and greater physical functioning (mean score $86.3 v 82.2, \mathrm{p}=0.04$ ) than the control group. The education group also had better scores than the peer discussion group on the mental health (mean score 73.4 $v 69.5, \mathrm{p}=0.04$ ), vitality (mean score $62.7 v 55.6, \mathrm{p}=0.00$ ), and social functioning (mean score $88.1 v 80.1, \mathrm{p}=0.00$ ) subscales of the SF-36.

\section{Conclusions}

In women with breast cancer, the effects of an education intervention were maintained at 3 years of follow up. The peer discussion intervention did not have a beneficial effect.

\section{COMMENTARY} psychosocial and physical quality of life indicators. necessity to meet numerous "hard to satisfy assumptions". studies. treatment.
Source of funding: National Institutes of Health.

For correspondence: Dr V S Helgeson Carnegie Mellon University, Pittsburgh, PA, USA.

vh2e@andrew.cmu.edu

The study by Helgeson et al contributes to the research on psychoeducational interventions for women with breast cancer. An abundance of research exists on the informational and emotional needs of these women, but little has been done to evaluate interventions to address these needs. It is unique to find a study that addresses the effect of psychosocial and educational interventions, separately and combined, in the same randomised controlled trial. It is also unique in that few studies have such a long follow up. Further strengths include a randomised, controlled design; a large sample that facilitates detection of between group differences; a similar number of participants randomised to each group; and a valid, reliable outcome measure that assesses both

A description of how randomisation was done and whether data collectors were blinded to group assignment would have helped readers determine whether a potential for bias existed. As the authors note, the effects are small and may not be generalisable to women of other ethnic backgrounds or those with lower levels of education or advanced disease. The use of repeated measures analysis of variance (ANOVA), although appropriate, seems unnecessarily cumbersome. A more interesting approach would have been to use individual slope analysis. This is an excellent methodology to analyse change over time, but is less constrained than repeated measures ANOVA by the

One drawback of existing intervention studies is that the outcome variables differ widely. The instrument chosen by Helgeson et al encompasses a broad range of physical and psychosocial factors that give a balanced picture of participants' quality of life. Its wider use would allow other researchers to examine quality of life outcomes of interest to them and compare the effect of their interventions to those of other

The findings indicate that an educational intervention given at the time of treatment may affect long term adjustment and quality of life among breast cancer survivors. Long term follow up provides an opportunity to examine how the effect of the intervention changes over time. For example, Helgeson et al found that improvement in vitality in the education group peaked 10 months after the study began, stayed the same at 16 months, dropped slightly at 28 months, and remained about the same at 40 months. This finding emphasises the need for longer term evaluation of psychoeducational interventions than has been the norm. The next step should be to test interventions that are more specifically matched to the information needs of patients at different stages of illness and

Carolyn Ingram, RN, CON(C), DNSc Assistant Professor, School of Nursing McMaster University Hamilton, Ontario, Canada

1 Knapp TR. Quantitative nursing research. Thousand Oaks, CA: Sage Publications, 1998 